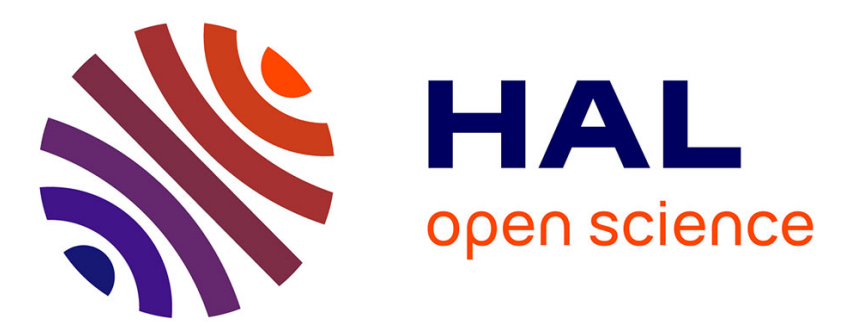

\title{
Operator-Norm Convergence of the Trotter Product Formula on Hilbert and Banach Spaces: A Short Survey
}

\author{
Hagen A Neidhardt, Artur A Stephan, Valentin A Zagrebnov
}

\section{To cite this version:}

Hagen A Neidhardt, Artur A Stephan, Valentin A Zagrebnov. Operator-Norm Convergence of the Trotter Product Formula on Hilbert and Banach Spaces: A Short Survey. Themistocles M. Rassias. Current Research in Nonlinear Analysis, 135, Springer, pp.229-247, 2018, Springer Optimization and Its Applications, 978-3-319-89799-8. 10.1007/978-3-319-89800-1_9 . hal-01971597

\section{HAL Id: hal-01971597 https://hal.science/hal-01971597}

Submitted on 11 Feb 2020

HAL is a multi-disciplinary open access archive for the deposit and dissemination of scientific research documents, whether they are published or not. The documents may come from teaching and research institutions in France or abroad, or from public or private research centers.
L'archive ouverte pluridisciplinaire $\mathbf{H A L}$, est destinée au dépôt et à la diffusion de documents scientifiques de niveau recherche, publiés ou non, émanant des établissements d'enseignement et de recherche français ou étrangers, des laboratoires publics ou privés. 


\section{Operator-norm convergence of the Trotter product formula on Hilbert and Banach spaces: a short survey}

Hagen Neidhardt, Artur Stephan and Valentin A. Zagrebnov

Dedicated to Haim Brezis and Louis Nirenberg un deep admiration

Abstract We give a review of results on the operator-norm convergence of the Trotter product formula on Hilbert and Banach spaces, which is focused on the problem of its convergence rates. Some recent results concerning evolution semigroups are presented in details.

\section{Introduction}

Recall that the product formula

$$
e^{-\tau C}=\lim _{n \rightarrow \infty}\left(e^{-\tau A / n} e^{-\tau B / n}\right)^{n}, \quad \tau \geq 0
$$

was established by $\mathrm{S}$. Lie (in 1875) for matrices where $C:=A+B$. The proof of formula (1.1) can be carried over easily to bounded operators on Banach spaces. Moreover, a straightforward computation shows that the convergence rate is $O(1 / n)$, i.e.

$$
\sup _{\tau \in[0, T]}\left\|e^{-\tau A / n} e^{-\tau B / n}-e^{-\tau C / n}\right\|=O(1 / n) .
$$

H. Trotter [25] has extended this result to unbounded operators $A$ and $B$ on Banach spaces, but in the strong operator topology. He proved that if $A$ and $B$ are generators of contractions semigroups on a separable Banach space such that the algebraic sum $A+B$ is a densely defined closable operator and the closure $C=\overline{A+B}$ is a generator of a contraction semigroup, then

H. Neidhardt

WIAS Berlin, Mohrenstr. 39, D-10117 Berlin, Germany

e-mail: hagen.neidhardt@wias-berlin.de

A. Stephan

HU Berlin, Institut für Mathematik, Unter den Linden 6, D-10099 Berlin, Germany

e-mail: stephan@math.hu-berlin.de

V.A. Zagrebnov

Université d'Aix-Marseille - Institut de Mathématiques de Marseille (UMR 7373), CMI-

Technopôle Château-Gombert, 39, rue F. Joliot Curie, 13453 Marseille

e-mail: valentin.zagrebnov@univ-amu.fr 


$$
e^{-\tau C}=\mathrm{s}-\lim _{n \rightarrow \infty}\left(e^{-\tau A / n} e^{-\tau B / n}\right)^{n}
$$

uniformly in $\tau \in[0, T]$ for any $T>0$.

Formula (1.3) is often called the Trotter or the Lie-Trotter product formula. It was a long-time belief that this formula is valid only in the strong operator topology. But in nineties it was discovered that under certain quite standard assumptions the strong convergence of the Trotter product formula can be improved to the operator-norm convergence. In the following we give a review of these results.

The paper is organised as follows. In Section 2.1 we give an overview on operator-norm convergence of the Trotter product formula if the generators $A$ and $B$ are non-negative self-adjoint operators. Section 2.2 summarises the case when one of the generator is only a maximal accretive operator. Section 2.3 is devoted to the evolution case, which arises in the theory of the abstract non-autonomous Cauchy problem. These results are commented in Section 2.4.

Section 3 is concerned with the operator-norm convergence of the Trotter product formula on the Banach spaces. Section 3.1 presents the results under the assumption that one of generators is for holomorphic semigroup. Section 3.2 considers again the evolution case but on Banach spaces. The relation between evolution semigroups and propagators is explained in Section 3.3. We comment the results in Section 3.4.

In Section 4 we collect some examples and counterexamples. They show what is expectable and what is not and even surprising.

We use below the following notations and definitions.

1. We use a definition of the semigroup generator $C$ (1.3), which differs from the standard one by a minus, as it is in the book [11].

2. Furthermore, we widely use the so-called Landau symbols:

$$
\begin{aligned}
& g(n)=O(f(n)) \Longleftrightarrow \limsup _{n \rightarrow \infty}\left|\frac{g(n)}{f(n)}\right|<\infty, \\
& g(n)=o(f(n)) \Longleftrightarrow \limsup _{n \rightarrow \infty}\left|\frac{g(n)}{f(n)}\right|=0, \\
& g(n)=\Theta(f(n)) \Longleftrightarrow 0<\liminf _{n \rightarrow \infty}\left|\frac{g(n)}{f(n)}\right| \leq \limsup _{n \rightarrow \infty}\left|\frac{g(n)}{f(n)}\right|<\infty, \\
& g(n)=\omega(f(n)) \Longleftrightarrow \limsup _{n \rightarrow \infty}\left|\frac{g(n)}{f(n)}\right|=\infty .
\end{aligned}
$$

3. We use the notation $C^{0, \beta}([0, T])$ for the Hölder $(\beta \in(0,1))$ and, respectively, for the Lipschitz $(\beta=1)$ continuous functions. 


\section{Trotter product formula on Hilbert spaces}

\subsection{Self-adjoint case}

Considering the Trotter product formula on a separable Hilbert space $\mathfrak{H}$ T. Kato has shown in $[9,10]$ that for non-negative operators $A$ and $B$ the Trotter formula (1.3) holds in the strong operator topology if $\operatorname{dom}(\sqrt{A}) \cap \operatorname{dom}(\sqrt{B})$ is dense in the Hilbert space and $C=A \dot{+} B$ is the form-sum of operators $A$ and $B$.

Naturally the problem arises whether Kato's result can be extended to the operator-norm convergence. A first attempt in this direction was undertaken by Rogava [23]. He claimed that if $A$ and $B$ are non-negative self-adjoint operators such that $\operatorname{dom}(A) \subseteq \operatorname{dom}(B)$ and the operator-sum: $C=A+B$, is self-adjoint, then

$$
\left\|\left(e^{-\tau A / n} e^{-\tau B / n}\right)^{n}-e^{-\tau C}\right\|=O(\ln (n) / \sqrt{n}), \quad n \rightarrow \infty,
$$

holds. In [15] it was shown that if one substitutes in above conditions the selfadjointness of the operator-sum by the $A$-smallness of $B$ with a relative bound less then one, then (2.4) is true with the rate of convergence improved to

$$
\left\|\left(e^{-\tau A / n} e^{-\tau B / n}\right)^{n}-e^{-\tau C}\right\|=O(\ln (n) / n), \quad n \rightarrow \infty
$$

The problem in its original formulation was finally solved in [8]. There it was shown that the best possible in this general setup rate (1.2) holds if the operator sum: $C=A+B$, is already a self-adjoint operator. Obviously, Rogava's result, as well as many other results (including [15]), when the operator sum of generators is self-adjoint, follow from [8] .

A new direction comes due to results for the fractional-power conditions. In [20], with elucidation in [6], it was proven that assuming

$$
\operatorname{dom}\left(C^{\alpha}\right) \subseteq \operatorname{dom}\left(A^{\alpha}\right) \cap \operatorname{dom}\left(B^{\alpha}\right), \quad \alpha \in(1 / 2,1), \quad C=A \dot{+} B,
$$

and

$$
\operatorname{dom}\left(A^{1 / 2}\right) \subseteq \operatorname{dom}\left(B^{1 / 2}\right)
$$

one obtains that

$$
\sup _{\tau \in[0, T]}\left\|\left(e^{-\tau A / n} e^{-\tau B / n}\right)^{n}-e^{-\tau C}\right\|=O\left(n^{-(2 \alpha-1)}\right) .
$$

Notice that formally $\alpha=1$ yields the rate obtained in [8].

We remark also that the results of $[6,20]$ do not cover the case $\alpha=1 / 2$. Although, it turns out that in this case the Trotter product formula converges on the operator norm:

$$
\sup _{\tau \in[0, T]}\left\|\left(e^{-\tau A / n} e^{-\tau B / n}\right)^{n}-e^{-\tau C}\right\|=o(1)
$$


if $\sqrt{B}$ is relatively compact with respect to $\sqrt{A}$, i.e. $\sqrt{B}(I+A)^{-1 / 2}$ is compact, see [16].

\subsection{Nonself-adjoint case}

Another direction was related with attempts to extend the the Trotter, and the Trotter-Kato, product formulae to the case of nonself-adjoint sectorial generators [3]. Let $A$ be a non-negative self-adjoint operator and let $B$ be a maximal accretive $(\Re e(B f, f) \geq 0$ for $f \in \operatorname{dom}(B)$ ) operator, such that

$$
\operatorname{dom}(A) \subseteq \operatorname{dom}(B) \quad \text { and } \quad \operatorname{dom}(A) \subseteq \operatorname{dom}\left(B^{*}\right) .
$$

If $B$ is $A$-small with a relative bound less than one, then the rate estimate (2.4) holds, for generator $C$, which is the well-defined maximal accretive operator-sum: $C=A+B$, see [1].

In [2] this result was generalised as follows. Let $A$ be a non-negative self-adjoint operator and let $B$ be a maximal accretive operator such that $\operatorname{dom}(A) \subseteq \operatorname{dom}(B)$ and $B$ is $A$-small with relative bound less than one. If the condition

$$
\operatorname{dom}\left(\left(C^{*}\right)^{\alpha}\right) \subseteq \operatorname{dom}\left(A^{\alpha}\right) \cap \operatorname{dom}\left(\left(B^{*}\right)^{\alpha}\right), \quad C=A+B,
$$

is satisfied for some $\alpha \in(0,1]$, then the norm-convergent Trotter product formula:

$$
\sup _{\tau \in[0, T]}\left\|\left(e^{-\tau A / n} e^{-\tau B / n}\right)^{n}-e^{-\tau C}\right\|=O\left(\ln (n) / n^{\alpha}\right),
$$

holds as $n \rightarrow \infty$.

In fact, more results are known about the operator-norm Trotter product formula convergence for nonself-adjoint semigroups, but without the rate estimates, see [4].

\subsection{Evolution case}

At the first glance a very different result about a Trotter-type product formula was obtained in [7]. The authors consider instead of the self-adjoint operator $B$ a family $\{B(t)\}_{t \in[0, T]}$ of self-adjoint operators on the separable Hilbert space $\mathfrak{H}$ such that the condition

$$
\operatorname{dom}\left(A^{\alpha}\right) \subseteq \operatorname{dom}(B(t)), \quad t \in[0, T]
$$

is satisfied for some $\alpha \in[0,1)$ for $A$, which is a non-negative self-adjoint operator. Then the operator sum $C(t)=A+B(t)$ defines a family of self-adjoint operators in $\mathfrak{H}$ space such that $\operatorname{dom}(C(t))=\operatorname{dom}(A), t \in[0, T]$. With the family $\{C(t)\}_{t \in[0, T]}$ one associates the evolution equation: 


$$
\partial_{t} u(t)=-C(t) u(t), \quad t \in[0, T],
$$

corresponding to the non-autonomous Cauchy problem with initial condition $u_{0}=$ $u(0)$ for $t=0$.

It turns out that equation (2.8) admits a propagator $\{U(t, s)\}_{(t, s) \in \bar{\Delta}}, \bar{\Delta}=\{(t, s) \in$ $[0, T] \times[0, T]: 0 \leq s \leq t \leq T\}$, which solves this problem. We remind that a family $\{U(t, s)\}_{(t, s) \in \Delta}$ of bounded operators is called a propagator if the operator-valued function $U(\cdot, \cdot): \bar{\Delta} \longrightarrow \mathscr{B}(\mathfrak{H})$ is strongly continuous and verifies the conditions:

$$
\begin{aligned}
& U(t, t)=I \quad \text { for } \quad t \in[0, T] \\
& U(t, s)=U(t, r) U(r, s) \text { for } \quad t, r, s \in[0, T] \quad \text { with } \quad s \leq r \leq t .
\end{aligned}
$$

Let $\left\{t_{j}\right\}_{j=0}^{N}$ be a partition of the closed interval $[0, t]$ :

$$
0=t_{0}<t_{1}<\ldots<t_{N-1}<t_{N}=t, \quad t_{j}=\tau j, \tau=t / N,
$$

for any $0<t \leq T$. Further, let

$$
Q_{j}(t, s ; n)=e^{-\frac{t-s}{n} A} e^{-\frac{t-s}{n} B\left(s+j \frac{t-s}{n}\right)}, \quad j=0,1, \ldots, n,
$$

and

$$
\begin{aligned}
E(t, s ; n) & :=Q_{n-1}(t, s ; n) Q_{n-2}(t, s ; n) \times \cdots \times Q_{1}(t, s ; n) Q_{0}(t, s ; n) \\
& :=\prod_{j=0}^{(n-1) \leftarrow} Q_{j}(t, s ; n)
\end{aligned}
$$

where the symbol $\prod_{j=1}^{n \leftarrow}$ means that the product is increasingly ordered in $j$ from the right to the left. If in addition to assumption (2.7), the condition

$$
\left\|A^{-\alpha}(B(t)-B(s)) A^{-\alpha}\right\| \leq L_{1}|t-s|, \quad t, s \in[0, T], \quad L_{1}>0,
$$

is satisfied, then in [7] it was proved that the propagator $\{U(t, s)\}_{(t, s) \in \bar{\Delta}}$, which solves the Cauchy problem (2.8), admits the approximation

$$
\sup _{t \in[0, T]}\left\|U(t, 0)-E_{n}(t, 0 ; n)\right\|=O(\ln (n) / n) \quad \text { as } \quad n \rightarrow \infty .
$$

Scrutinising the proof in [7] one finds that in fact the claim (2.14) can be slightly generalised to any interval $\bar{\Delta}$

$$
\sup _{(t, s) \in \bar{\Delta}}\left\|U(t, s)-E_{n}(t, s ; n)\right\|=O(\ln (n) / n) \quad \text { as } \quad n \rightarrow \infty .
$$

At the first glance, it seems that the result (2.15) is quite far from the Trotter product formula. However, this is not the case. To show this we follow the evolution semigroup approach to evolution equations developed in [12, 21]. Let us introduce the Hilbert space $L^{2}([0, T], \mathfrak{H})$ and consider on this space the semigroup 


$$
(\mathscr{U}(\tau) f)(t):=U(t, t-\tau) \chi_{[0, T]}(t-\tau) f(t-\tau), \quad f \in L^{2}([0, T], \mathfrak{H}),
$$

$t \in[0, T]$. It turns out that $\{\mathscr{U}(\tau)\}_{\tau \in \mathbb{R}_{+}}$is a $C_{0}$-semigroup on the Hilbert space of the $\mathfrak{H}$-valued trajectories $L^{2}([0, T], \mathfrak{H})$. By $\mathscr{K}$ we denote its generator, then $\mathscr{U}(\tau)=$ $e^{-\tau \mathscr{K}}, \tau \geq 0$.

Let us introduce two multiplication operators

$$
\begin{aligned}
(\mathscr{A} f)(t) & =A f(t), \quad f \in \operatorname{dom}(\mathscr{A}), \\
\operatorname{dom}(\mathscr{A}) & :=\left\{\begin{array}{c}
\left.f \in L^{2}([0, T], \mathfrak{H}): \begin{array}{c}
f(t) \in \operatorname{dom}(A) \quad \text { for a.e. } t \in[0, T] \\
A f(t) \in L^{2}([0, T], \mathfrak{H})
\end{array}\right\},
\end{array}\right.
\end{aligned}
$$

and

$$
\begin{aligned}
(\mathscr{B} f)(t) & =B(t) f(t), \quad f \in \operatorname{dom}(\mathscr{B}), \\
\operatorname{dom}(\mathscr{B}) & :=\left\{f \in L^{2}([0, T], \mathfrak{H}): \begin{array}{c}
f(t) \in \operatorname{dom}(B(t)) \quad \text { for a.e. } t \in[0, T] \\
B(t) f(t) \in L^{2}([0, T], \mathfrak{H})
\end{array}\right\} .
\end{aligned}
$$

Note that both operators are well-defined and self-adjoint in $L^{2}([0, T], \mathfrak{H})$. Moreover, condition (2.7) yields: $\operatorname{dom}(\mathscr{A}) \subseteq \operatorname{dom}(\mathscr{B})$.

By $D_{0}$ we define in $L^{2}([0, T], \overline{\mathfrak{H}})$ the generator of the right-shift semigroup $e^{-\tau D_{0}}$ given by

$$
\left(e^{-\tau D_{0}} f\right)(t)=\chi_{[0, T]}(t-\tau) f(t-\tau), \quad f \in L^{2}([0, T], \mathfrak{H}) .
$$

Notice that the operator $D_{0}$ is defined by

$$
\begin{aligned}
\left(D_{0} f\right)(t) & =\frac{\partial}{\partial t} f(t), \\
f \in \operatorname{dom}\left(D_{0}\right) & :=\left\{f \in W^{2,2}([0, T], \mathfrak{H}): f(0)=0\right\} .
\end{aligned}
$$

Collecting these definitions we introduce the operator

$$
\begin{aligned}
(\widetilde{\mathscr{K}} f) & =D_{0} f+\mathscr{A} f+\mathscr{B} f \\
f \in \operatorname{dom}(\widetilde{\mathscr{K}}) & :=\operatorname{dom}\left(D_{0}\right) \cap \operatorname{dom}(\mathscr{A}) \cap \operatorname{dom}(\mathscr{B}) .
\end{aligned}
$$

Since $\{U(t, s)\}_{(t, s) \in \bar{\Delta}}$ is a propagator solving the evolution equation (2.8) one deduces that by virtue of assumptions (2.7) and (2.13) the operator $\widetilde{\mathscr{K}}$ is closable and that its closure coincides with generator $\mathscr{K}$, see [12, Theorem 4.5].

Furthermore, let us define the operator

$$
\left(\mathscr{K}_{0} f\right)(t)=D_{0} f+\mathscr{A} f, \quad f \in \operatorname{dom}\left(\mathscr{K}_{0}\right):=\operatorname{dom}\left(D_{0}\right) \cap \operatorname{dom}(\mathscr{A}) .
$$

Then operator $\mathscr{K}_{0}$ is a generator of the $C_{0}$-semigroup, which has the form

$$
\left(e^{-\tau \mathscr{K}_{0}} f\right)(t)=e^{-\tau A} \chi_{t \in[0, T]}(t-\tau) f(t-\tau), \quad f \in L^{2}([0, T], \mathfrak{H}) .
$$

Note that we obviously get that $\mathscr{K}=\overline{\mathscr{K}_{0}+\mathscr{B}}=\mathscr{K}_{0}+\mathscr{B}$. 
For the pair $\left\{\mathscr{K}_{0}, \mathscr{B}\right\}$ one obtains the following result.

Proposition 2.1 Let $A$ be a non-negative self-adjoint operator on the separable Hilbert space $\mathfrak{H}$ and let $\{B(t)\}_{t \in[0, T]}$ be a family of non-negative self-adjoint operators. If the assumptions (2.7) and (2.13) are satisfied, then

$$
\sup _{\tau \geq 0}\left\|\left(e^{-\tau \mathscr{K}_{0} / n} e^{-\tau \mathscr{B} / n}\right)^{n}-e^{-\tau \mathscr{K}}\right\|=O(\ln (n) / n) \quad \text { for } n \rightarrow \infty
$$

and

$$
\sup _{\tau \geq 0}\left\|\left(e^{-\tau \mathscr{B} / n} e^{-\tau \mathscr{K}_{0} / n}\right)^{n}-e^{-\tau \mathscr{K}}\right\|=O(\ln (n) / n) \quad \text { for } n \rightarrow \infty
$$

Proof. A straightforward computation shows that for $f \in L^{2}([0, T], \mathfrak{H})$ one gets

$$
\left(\left(e^{-\tau \mathscr{K}_{0} / n} e^{-\tau \mathscr{B} / n}\right)^{n} f\right)(t)=E(t, t-\tau ; n) \chi_{[0, T]}(t-\tau) f(t-\tau),
$$

where $E(t, s ; n)$ is given by (2.12). Taking into account (2.16) we obtain

$$
\begin{aligned}
& \left(\left(e^{-\tau \mathscr{K}_{0} / n} e^{-\tau \mathscr{B} / n}\right)^{n} f\right)(t)-\left(e^{-\tau \mathscr{K}} f\right)(t) \\
& \quad=(E(t, t-\tau ; n)-U(t, t-\tau)) \chi_{[0, T]}(t-\tau) f(t-\tau),
\end{aligned}
$$

which yields the estimate

$$
\begin{aligned}
\left\|\left(\left(e^{-\tau \mathscr{K}_{0} / n} e^{-\tau \mathscr{B} / n}\right)^{n} f\right)(t)-\left(e^{-\tau \mathscr{K}} f\right)(t)\right\| \\
\quad \leq \chi_{[0, T]}(t-\tau)\|E(t, t-\tau ; n)-U(t, t-\tau)\|\|f(t-\tau)\| .
\end{aligned}
$$

Hence, this implies

$$
\begin{aligned}
\sup _{\tau \geq 0} \| & \left(e^{-\tau \mathscr{K}_{0} / n} e^{-\tau \mathscr{B} / n}\right)^{n} f-e^{-\tau \mathscr{K}} f \|^{2} \\
& \leq \sup _{\tau \geq 0} \int_{0}^{T} \chi_{[0, T]}(t-\tau)\|E(t, t-\tau ; n)-U(t, t-\tau)\|^{2}\|f(t-\tau)\|^{2} d t \\
& \leq \int_{0}^{T} \sup _{(t, s) \in \bar{\Delta}}\|E(t, s ; n)-U(t, s)\|^{2}\|f(r)\|^{2} d r .
\end{aligned}
$$

Using (2.15) we immediately obtain (2.20). Similarly (2.21) follows from [7].

\subsection{Comments}

Section 2.1

The operator-norm convergence rate $O(1 / n)$ of [8] for pairs of non-negative selfadjoint operators is sharp and ultimate optimal due to observations in [24]. The same remark concerns the sharpness and optimality of the rate $O\left(1 / n^{2 \alpha-1}\right)$ obtained first 
in [20] under assumption (2.5) together with the $A^{\alpha}$-smallness of $B^{\alpha}$ with relative bound less then one. Then the same ultimate sharp rate was proven in [6], when the smallness condition is relaxed to the mild subordination (2.6). It is an open problem whether the assumption (2.6) is really necessary.

\section{Section 2.2}

It is unclear whether the convergence rates $O(\ln (n) / n)$ and $O\left(\ln (n) / n^{\alpha}\right)$ are sharp. One expects convergence rates identical to that in Section 2.1.

Section 2.3

The approach used here was developed in $[12,17,18,19,21,22]$. The idea is to transform a time-dependent evolution problem to a time-independent problem, see also the next section.

Let us add some remarks. One easily checks that the operator $\mathscr{K}_{0}$ is not selfadjoint whereas the operator $\mathscr{B}$ is self-adjoint. However, $\mathscr{K}_{0}$ is maximal accretive. This is in some sense in contrast to Section 2.2, where the pair $\{A, B\}$ consists of a self-adjoint operator $A$ and an maximal accretive operator $B$ such that $\operatorname{dom}(A) \subseteq \operatorname{dom}(B)$ and $\operatorname{dom}(A) \subseteq \operatorname{dom}\left(B^{*}\right)$. In the evolution case the conditions $\operatorname{dom}\left(\mathscr{K}_{0}\right) \subseteq \operatorname{dom}(\mathscr{B})$ and $\operatorname{dom}\left(\mathscr{K}_{0}^{*}\right) \subseteq \operatorname{dom}(\mathscr{B})$ are satisfied but in the reversed order with respect to Section 2.2. However, the convergence rate $O(\ln (n) / n)$ is not affected by this.

The proof of the estimate (2.14) and (2.15) are very involved. Naturally the problem arises whether one can give a direct proof the estimate (2.14) avoiding those propagator estimates.

\section{Trotter product formula on Banach spaces}

\subsection{Holomorphic case}

There are only few generalisations of the results of Section 2 to Banach spaces. The main obstacle for that is the fact that the concept of self-adjointness is missing in the Banach spaces. One of solution is to relax the self-adjointness replacing the non-negative self-adjoint generator $A$ by a generator of the holomorphic semigroup. The following result was proved in [5].

Theorem 3.1 ([5, Theorem 3.6 and Corollary 3.7]) Let A be a generator of a holomorphic contraction semigroup on the separable Banach space $\mathfrak{X}$ and let $B$ a generator of a contraction semigroup on $\mathfrak{X}$.

(i) If for some $\alpha \in(0,1)$ the condition

$$
\operatorname{dom}\left(A^{\alpha}\right) \subseteq \operatorname{dom}(B),
$$

holds and $\operatorname{dom}\left(A^{*}\right) \subseteq \operatorname{dom}\left(B^{*}\right)$ is satisfied, then the operator sum $C=A+B$ is a generator of a contraction semigroup and 


$$
\sup _{\tau \in[0, T]}\left\|\left(e^{-\tau B / n} e^{-\tau A / n}\right)^{n}-e^{-\tau C}\right\|=O\left(\ln (n) / n^{1-\alpha}\right),
$$

for any $T>0$.

(ii) If for some $\alpha \in(0,1)$ the condition

$$
\operatorname{dom}\left(\left(A^{\alpha}\right)^{*}\right) \subseteq \operatorname{dom}\left(B^{*}\right),
$$

is satisfied and $\operatorname{dom}(A) \subseteq \operatorname{dom}(B)$ is valid, then $C=A+B$ is the generator of a contraction semigroup and

$$
\sup _{\tau \in[0, T]}\left\|\left(e^{-\tau A / n} e^{-\tau B / n}\right)^{n}-e^{-\tau C}\right\|=O\left(\ln (n) / n^{1-\alpha}\right),
$$

for any $T>0$.

Theorem 3.2 ([5, Theorem 3.6 and Corollary 3.7]) Let A be a generator of a holomorphic contraction semigroup on $\mathfrak{X}$ and let $B$ a generator of a contraction semigroup on $\mathfrak{X}$. If $B$ is in addition a bounded operator, then

$$
\sup _{\tau \in[0, T]}\left\|\left(e^{-\tau B / n} e^{-\tau A / n}\right)^{n}-e^{-\tau C}\right\|=O\left((\ln (n))^{2} / n\right),
$$

and

$$
\sup _{\tau \in[0, T]}\left\|\left(e^{-\tau A / n} e^{-\tau B / n}\right)^{n}-e^{-\tau C}\right\|=O\left((\ln (n))^{2} / n\right),
$$

for any $T>0$.

\subsection{Evolution case}

Similarly of Section 2.3 let us consider a generator $A$ of a holomorphic semigroup on the separable Banach space $\mathfrak{X}$ and a family of $\{B(t)\}_{t \in[0, T]}$ of generators of holomorphic semigroups on $\mathfrak{X}$. We make the following assumptions:

\section{Assumption 3.3}

(A1) The operator $A$ is a generator of a holomorphic contraction semigroup on $\mathfrak{X}$ such that $0 \in \rho(A)$.

(A2) Let $\{B(t)\}_{t \in[0, T]}$ be a family of closed operators such that for a.e. $t \in[0, T]$ and some $\alpha \in(0,1)$ the condition $\operatorname{dom}\left(A^{\alpha}\right) \subset \operatorname{dom}(B(t))$ is satisfied such that

$$
C_{\alpha}:=\operatorname{ess}_{\sup _{t \in[0, T]}}\left\|B(t) A^{-\alpha}\right\|_{\mathscr{B}(X)}<\infty .
$$

(A3) Let $\{B(t)\}_{t \in[0, T]}$ be a family of generators of contraction semigroups in $\mathfrak{X}$ such that the function $[0, T] \ni t \mapsto(B(t)+\xi)^{-1} x \in \mathfrak{X}$ is strongly measurable for any $x \in \mathfrak{X}$ and any $\xi>b>0$.

(A4) We assume that $\operatorname{dom}\left(A^{*}\right) \subset \operatorname{dom}\left(B(t)^{*}\right)$ and 


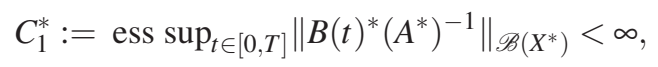

where $A^{*}$ and $B(t)^{*}$ denote operators which are adjoint of $A$ and $B(t)$, respectively. (A5) There exists $\beta \in(\alpha, 1)$ and a constant $L_{\beta}>0$ such that for a.e. $t, s \in[0, T]$ one has the estimate:

$$
\left\|A^{-1}(B(t)-B(s)) A^{-\alpha}\right\| \leq L_{\beta}|t-s|^{\beta} .
$$

(A6) There exists a constant $L_{1}>0$ such that for a.e. $t, s \in[0, T]$ one has the estimate:

$$
\left\|A^{-\alpha}(B(t)-B(s)) A^{-\alpha}\right\| \leq L_{1}|t-s| .
$$

The assumption $0 \in \rho(A)$ in (A1) is made for simplicity. We note that assumption (A3) is similar to (2.7). Assumption (A4) is automatically satisfied for self-adjoint operators. Assumption (A5) is a modification of (2.13) while assumption (A6) coincides with (2.13).

With the family $\{C(t)\}_{t \in[0, T]}, C(t)=A+B(t)$, one associates the evolution equation (2.8). It turns out that under the assumptions (A1) and (A2) the family $\{C(t)\}_{t \in[0, T]}$ consists of generators of contraction semigroups.

In accordance with Section 2.3 we consider the Banach space $L^{p}([0, T], \mathfrak{X})$ for some fixed $p \in[0,1)$ and introduce the multiplication operators $\mathscr{A}$ and $\mathscr{B}$, cf. (2.17) and (2.18). Under the assumptions (A1)-(A3) the operators $\mathscr{A}$ and $\mathscr{B}$ are generators of contraction semigroups such that $\operatorname{dom}(\mathscr{A}) \subseteq \operatorname{dom}(\mathscr{B})$, in particular, $\mathscr{A}$ is a generator of holomorphic semigroup. Similarly, one can introduce the multiplication operator $\mathscr{C}$ induced by the family $\{C(t)\}_{t \in[0, T]}$ which is also a generator of a holomorphic semigroup. Notice that $\mathscr{C}=\mathscr{A}+\mathscr{B}$ and $\operatorname{dom}(\mathscr{C})=\operatorname{dom}(\mathscr{A})$.

Let $D_{0}$ the generator of the right-shift semigroup on $L^{p}([0, T], \mathfrak{X})$, i.e.

$$
\left(e^{-\tau D_{0}} f\right)(t)=f(t-\tau) \chi_{[0, T]}(t-\tau) f(t-\tau), \quad f \in L^{p}([0, T], \mathfrak{X}),
$$

cf. (2.19). We consider the operator

$$
\begin{aligned}
\widetilde{\mathscr{K}} f & =D_{0} f+\mathscr{A} f+\mathscr{B} f, \\
f \in \operatorname{dom}(\widetilde{\mathscr{K}}) & =\operatorname{dom}\left(D_{0}\right) \cap \operatorname{dom}(\mathscr{A}) \cap \operatorname{dom}(\mathscr{B}),
\end{aligned}
$$

cf. (2.19). Assuming (A1)-(A3) it was shown in [12] that the operator $\widetilde{K}$ is closable and its closure $\mathscr{K}$ is the generator of a semigroup.

Furthermore, we set

$$
\widetilde{\mathscr{K}_{0}} f=D_{0} f+\mathscr{A} f, \quad f \in \operatorname{dom}\left(\widetilde{\mathscr{K}_{0}}\right)=\operatorname{dom}\left(D_{0}\right) \cap \operatorname{dom}(\mathscr{A}),
$$

In contrast to the Hilbert space the operator $\widetilde{\mathscr{K}_{0}}$ is not necessary a generator of a semigroup. However, the operator $\mathscr{K}_{0}$ closable and its closure $\mathscr{K}_{0}$ is a generator. Notice that $\mathscr{K}$ coincides with the algebraic sum of $\mathscr{K}_{0}$ and $\mathscr{B}$, i.e $\mathscr{K}=\mathscr{K}_{0}+\mathscr{B}$.

In [12] the following theorem was proved. 
Theorem 3.4 ([12, Theorem 7.8]) Let the assumptions (A1)-(A4) be satisfied for some $\alpha \in(0,1)$. If (A5) holds, then

$$
\sup _{\tau \geq 0}\left\|\left(e^{-\tau B / n} e^{-\tau \mathscr{K}_{0} / n}\right)^{n}-e^{-\tau \mathscr{K}}\right\|=O\left(1 / n^{\beta-\alpha}\right),
$$

when $n \rightarrow \infty$.

Assuming instead of assumption (A5) the assumption (A6) the result slightly modifies, see [14].

Theorem 3.5 ([14, Theorem 5.4]) Let the assumptions (A1)-(A4) be satisfied for some $\alpha \in(1 / 2,1)$. If (A6) is valid, then for $n \rightarrow \infty$ one gets the asymptotic:

$$
\sup _{\tau \geq 0}\left\|\left(e^{-\tau B / n} e^{-\tau \mathscr{K} 0 / n}\right)^{n}-e^{-\tau \mathscr{K}}\right\|=O\left(1 / n^{1-\alpha}\right) .
$$

\subsection{Convergence rates for propagators}

The proof of both theorems does not use propagator approximations of type (2.14) or (2.15). However, Theorem 3.4 and 3.5 can be used to prove propagator approximations. To this end one has to introduce the notion of a evolution semigroup.

Definition 3.6 A generator $\mathscr{K}$ in $L^{p}([0, T], X), p \in[1, \infty)$, is called a evolution generator if

(i) $\operatorname{dom}(\mathscr{K}) \subset C([0, T], X)$ and $M(\phi) \operatorname{dom}(\mathscr{K}) \subset \operatorname{dom}(\mathscr{K})$ for $\phi \in W^{1, \infty}([0, T])$,

(ii) $\mathscr{K} M(\phi) f-M(\phi) \mathscr{K} f=M(\dot{\phi})$ f for $f \in \operatorname{dom}(\mathscr{K})$ and $\phi \in W^{1, \infty}([0, T])$, where $\dot{\phi}=\partial_{t} \phi$,

(iii) the domain $\operatorname{dom}(\mathscr{K})$ has a dense cross-section, i.e. for each $t \in(0, T]$ the set

$$
[\operatorname{dom}(\mathscr{K})]_{t}:=\{x \in \mathfrak{X}: \exists f \in \operatorname{dom}(\mathscr{K}) \text { such that } x \in f(t)\},
$$

is dense in $\mathfrak{X}$.

By $M(\phi), L^{\infty}([0, T])$, the bounded operator

$$
(M(\phi) f)(t)=\phi(t) f(t), \quad f \in L^{p}([0, T], \mathfrak{X}) .
$$

is meant.

One can check that the operator $\mathscr{K}$ defined as the closure of $\widetilde{\mathscr{K}}$ is an evolution generator, cf. [12, Theorem 1.2]. Evolution generators a directly related to propagators. For this purpose one has slightly weaken the notion of a propagator defined in Section 2.3.

Definition 3.7 Let $\{U(t, s)\}_{(t, s) \in \Delta}, \Delta=\{(t, s) \in(0, T] \times(0, T]: s \leq t \leq T\}$, be a strongly continuous family of bounded operators on $\mathfrak{X}$. If the conditions 


$$
\begin{aligned}
& U(t, t)=I \text { for } t \in(0, T], \\
& U(t, r) U(r, s)=U(t, s) \text { for } t, r, s \in(0, T] \text { with } \quad s \leq r \leq t, \\
& \|U\|_{\mathscr{B}(X)}:=\sup _{(t, s) \in \Delta}\|U(t, s)\|<\infty
\end{aligned}
$$

are satisfied, then $\{U(t, s)\}_{(t, s) \in \Delta}$ is called a propagator.

Comparing with Section 2.3 we note that $\Delta$ slightly differs from $\bar{\Delta}$. Indeed, $\Delta \subseteq \bar{\Delta}$ but $\bar{\Delta}=\bar{\Delta}$. Restricting (2.9) and (2.10) to $(0, T]$ we get (3.26) and (3.27), respectively. Condition (3.28) is necessary because the set $\Delta$ is not closed.

It is known that there is an one-to-one correspondence between the set of all evolution generators on $L^{p}([0, T], \mathfrak{X})$ and the set of all propagators in the sense of Definition 3.7 established by

$$
\left(e^{-\tau \mathscr{K}} f\right)(t)=U(t, t-\tau) \chi_{[0, T]}(t-\tau) f(t-\tau), \quad f \in L^{p}([0, T], \mathfrak{X}),
$$

cf. [12, Theorem 3.3] or [17, Theorem 4.12].

Let $\mathscr{K}_{0}$ be the generator of an evolution semigroup $\left\{\mathscr{U}_{0}(\tau)\right\}_{\tau \geq 0}$ and let $\mathscr{B}$ be a multiplication operator induced by a measurable family $\{B(t)\}_{t \in[0, T]}$ of generators of contraction semigroups. Note that in this case the multiplication operator $\mathscr{B}$ is a generator of a contraction semigroup $\left(e^{-\tau \mathscr{B}} f\right)(t)=e^{-\tau B(t)} f(t)$, on the Banach space $L^{p}([0, T], X)$. Since $\left\{\mathscr{U}_{0}(\tau)\right\}_{\tau \geq 0}$ is an evolution semigroup, then there is a propagator $\left\{U_{0}(t, s)\right\}_{(t, s) \in \Delta}$ such that the representation

$$
\left(\mathscr{U}_{0}(\tau) f\right)(t)=U_{0}(t, t-\tau) \chi_{[0, T]}(t-\tau) f(t-\tau), \quad f \in L^{p}([0, T], X),
$$

is valid for a.e. $t \in[0, T]$ and $\tau \geq 0$. Then we define

$$
Q_{j}(t, s ; n):=U_{0}\left(s+j \frac{(t-s)}{n}, s+(j-1) \frac{(t-s)}{n}\right) e^{-\frac{(t-s)}{n} B\left(s+(j-1) \frac{(t-s)}{n}\right)}
$$

where $j \in\{1,2, \ldots, n\}, n \in \mathbb{N},(t, s) \in \Delta$, and we set

$$
V_{n}(t, s):=\prod_{j=1}^{n \leftarrow} Q_{j}(t, s ; n), \quad n \in \mathbb{N}, \quad(t, s) \in \Delta,
$$

where the product is increasingly ordered in $j$ from the right to the left. Then a straightforward computation shows that the representation

$$
\left(\left(e^{-\tau \mathscr{K}_{0} / n} e^{-\tau \mathscr{B} / n}\right)^{n} f\right)(t)=V_{n}(t, t-\tau) \chi_{[0, T]}(t-\tau) f(t-\tau),
$$

$f \in L^{p}([0, T], X)$, holds for each $\tau \geq 0$ and a.e. $t \in[0, T]$. Similarly we can introduce

$$
G_{j}(t, s ; n)=e^{-\frac{t-s}{n} B\left(s+j \frac{t-s}{n}\right)} U_{0}\left(s+j \frac{t-s}{n}, s+(j-1) \frac{t-s}{n}\right)
$$

where $j \in\{1,2, \ldots, n\}, n \in \mathbb{N},(t, s) \in \Delta$. Let 


$$
U_{n}(t, s):=\prod_{j=1}^{n \leftarrow} G_{j}(t, s ; n), \quad n \in \mathbb{N}, \quad(t, s) \in \Delta,
$$

where the product is again increasingly ordered in $j$ from the right to the left. We verify that

$$
\left(\left(e^{-\tau \mathscr{B} / n} e^{-\tau \mathscr{K} \mathscr{K}_{0} / n}\right)^{n} f\right)(t)=U_{n}(t, t-\tau) \chi_{[0, T]}(t-\tau) f(t-\tau),
$$

$f \in L^{p}([0, T], X)$, holds for each $\tau \geq 0$ and a.e. $t \in[0, T]$.

Proposition 3.8 ([13, Proposition 2.1]) Let $\mathscr{K}$ and $\mathscr{K}_{0}$ be generators of evolution semigroups on the Banach space $L^{p}([0, T], X)$ for some $p \in[1, \infty)$. Further, let $\{B(t))\}_{t \in[0, T]}$ be a strongly measurable family of generators of contraction on $\mathfrak{X}$. Then

$$
\sup _{\tau \geq 0}\left\|e^{-\tau \mathscr{K}}-\left(e^{-\tau \mathscr{K} 0 / n} e^{-\tau \mathscr{B} / n}\right)^{n}\right\|=\underset{(t, s) \in \Delta}{\operatorname{esssup}}\left\|U(t, s)-V_{n}(t, s)\right\|, \quad n \in \mathbb{N},
$$

and

$$
\sup _{\tau \geq 0}\left\|e^{-\tau \mathscr{K}}-\left(e^{-\tau \mathscr{B} / n} e^{-\tau \mathscr{K} 0 / n}\right)^{n}\right\|=\underset{(t, s) \in \Delta}{\operatorname{esssup}}\left\|U(t, s)-U_{n}(t, s)\right\|, \quad n \in \mathbb{N} .
$$

Let us introduce the approximations

$$
\begin{aligned}
U_{n}(t, s) & :=\prod_{j=1}^{n \leftarrow} G_{j}(t, s ; n), \quad n=1,2, \ldots, \\
G_{j}(t, s ; n) & :=e^{-\frac{t-s}{n} B\left(s+j \frac{t-s}{n}\right)} e^{-\frac{t-s}{n} A}, \quad j=0,1,2, \ldots, n,
\end{aligned}
$$

$(t, s) \in \Delta$, with increasingly ordered product in $j$ from the right to the left. From Theorem 3.4 and Proposition 3.8 one immediately obtains the following theorem.

Theorem 3.9 ([12, Theorem 1.4]) Let the assumptions (A1)-(A4) be satisfied. If (A5) holds, then for $n \rightarrow \infty$ the rate:

$$
\underset{(t, s) \in \Delta}{\operatorname{ess} \sup }\left\|U_{n}(t, s)-U(t, s)\right\|=O\left(1 / n^{\beta-\alpha}\right) .
$$

From Theorem 3.5 and Proposition 3.8 we get

Theorem 3.10 ([14, Theorem 5.6]) Let the assumptions (A1)-(A4) be satisfied for some $\alpha \in(1 / 2,1)$. If $(A 6)$ is valid, then for $n \rightarrow \infty$ one obtains a better rate:

$$
\underset{(t, s) \in \Delta}{\operatorname{ess} \sup }\left\|U_{n}(t, s)-U(t, s)\right\|=O\left(1 / n^{1-\alpha}\right) .
$$




\subsection{Comments}

\section{Section 3.1}

It is unclear whether Theorem 3.1 is sharp. Theorem 3.1 should be valid if the contractivity of the involved semigroups is replaced Trotter-stability. A pair of generators $\{A, B\}$ is called Trotter-stable if the Trotter product is uniformly bounded in $n \in \mathbb{N}$, i.e., if

$$
\sup _{n \in \mathbb{N}} \sup _{\tau \geq 0}\left\|\left(e^{-\tau A / n} e^{-\tau B / n}\right)^{n}\right\|<\infty .
$$

It turns out that the pair $\{A, B\}$ is Trotter-stable if and only $\{B, A\}$ is Trotter-stable.

\section{Section 3.2}

Theorems 3.4 and 3.5 are proved in [12] and [14] under the assumption that the pair $\left\{\mathscr{K}_{0}, \mathscr{B}\right\}$ is Trotter-stable. The convergence rates (3.24) and (3.25) differ significantly from the convergence rate $O(\ln (n) / n)$ of Proposition 2.1. It is an open problem whether the convergences rates (3.24) and (3.25) can be improved to $O(\ln (n) / n)$. One has to mention that the convergence (3.25) coincides with that one of (3.23) despite the fact that $\mathscr{K}_{0}$ is not a generator of a holomorphic semigroup. Indeed, the generator $\mathscr{K}_{0}$ is not holomorphic since $e^{-\tau \mathscr{K}_{0}}=0$ for $\tau \geq T$.

\section{Section 3.3}

It is a bit surprising that the operator-norm convergence of the Trotter product formula for the pairs: generator of evolution semigroup and multiplication operator, is equivalent to the operator-norm convergence of a certain approximation of the corresponding propagator, see Proposition 3.8. In particular, this yields that two convergences: (2.15) and (2.20), are equivalent.

\section{Sharpness}

\subsection{Example}

Let us consider a "solvable" example. We study bounded perturbations of the evolution generator $D_{0}$. To do this aim we consider $\mathfrak{X}=\mathbb{C}$ and we denote by $L^{2}([0,1])$ the Hilbert space $L^{2}([0, T], \mathbb{C})$.

For $t \in[0,1]$, let $q: t \mapsto q(t) \in L^{\infty}([0,1])$. Then, $q$ induces a bounded multiplication operator $Q$ in the Banach space $L^{2}([0,1])$ :

$$
(Q f)(t)=q(t) f(t), \quad f \in L^{2}([0,1]) .
$$

For simplicity we assume that $q \geq 0$. Then $Q$ generates on $L^{p}([0,1])$ a contraction semigroup $\left\{e^{-\tau Q}\right\}_{\tau \geq 0}$. Since generator $Q$ is bounded, the closed operator $\mathscr{K}:=D_{0}+Q$, with domain $\operatorname{dom}(\mathscr{K})=\operatorname{dom}\left(D_{0}\right)$, is generator of a semigroup on 
$L^{p}([0,1])$. From [25] one gets immediately

$$
\text { s- } \lim _{n \rightarrow \infty}\left(e^{-\tau D_{0} / n} e^{-\tau Q / n}\right)^{n}=e^{-\tau\left(D_{0}+Q\right)}
$$

uniformly in $\tau \in[0, T]$ for any $T>0$. One easily checks that $\mathscr{K}$ is an evolution generator. A straightforward computation shows that

$$
\left(e^{-\tau\left(D_{0}+Q\right)} f\right)(t)=e^{-\int_{t-\tau}^{t} q(y) d y} \chi_{[0,1]}(t-\tau) f(t-\tau)
$$

which yields that the propagator corresponding to $\mathscr{K}$ is given by

$$
U(t, s)=e^{-\int_{s}^{t} q(y) d y}, \quad(t, s) \in \Delta .
$$

A simple computation shows that

$$
\left(\left(e^{-\tau D_{0} / n} e^{-\tau Q / n}\right)^{n} f\right)(t)=: V_{n}(t, t-\tau) \chi_{[0, T]}(t-\tau) f(t-\tau) .
$$

Then by straightforward calculations one finds that

$$
V_{n}(t, s)=e^{-\frac{t-s}{n} \sum_{k=0}^{n-1} q\left(s+k \frac{t-s}{n}\right)}, \quad(t, s) \in \Delta .
$$

Proposition 4.1 ([13, Proposition 3.1]) Let $q \in L^{\infty}([0, T])$ be non-negative. Then

$$
\begin{aligned}
\sup _{\tau \geq 0}\left\|e^{-\tau\left(D_{0}+Q\right)}-\left(e^{-\tau D_{0} / n} e^{-\tau Q / n}\right)^{n}\right\|_{\mathscr{B}\left(L^{p}([0,1])\right)} \\
=\Theta\left(\operatorname{esssup}_{(t, s) \in \Delta}\left|\int_{s}^{t} q(y) d y-\frac{t-s}{n} \sum_{k=0}^{n-1} q\left(s+k \frac{t-s}{n}\right)\right|\right)
\end{aligned}
$$

as $n \rightarrow \infty$, where $\Theta$ is the Landau symbol defined in the introduction.

Note that by Proposition 3.8 the operator-norm convergence rate of the Trotter product formula for the pair $\left\{D_{0}, Q\right\}$ coincides with the convergence rate of the integral Darboux-Riemann sum approximation of the Lebesgue integral.

\subsection{Results}

Below we give a series of examples which show the dependence of the convergence rate on the smoothness of the function $q \in L^{\infty}([0, T])$. First we consider the Hölder and Lipschitz continuous cases.

Theorem 4.2 ([13, Theorem 3.2]) If the function: $q \in C^{0, \beta}([0, T]), \beta \in(0,1]$, is non-negative, then for $n \rightarrow \infty$ one gets 


$$
\sup _{\tau \geq 0}\left\|e^{-\tau\left(D_{0}+Q\right)}-\left(e^{-\tau D_{0} / n} e^{-\tau Q / n}\right)^{n}\right\|=O\left(1 / n^{\beta}\right)
$$

Now a natural question that one may to ask is: what happens, when $q$ is simply continuous?

Theorem 4.3 ([13, Theorem 3.3]) If $q:[0,1] \rightarrow \mathbb{C}$, is continuous and non-negative, then for $n \rightarrow \infty$

$$
\left\|e^{-\tau\left(D_{0}+Q\right)}-\left(e^{-\tau D_{0} / n} e^{-\tau Q / n}\right)^{n}\right\|=o(1) .
$$

We comment that for a general continuous $q$ one can say nothing about the Trotter product formula convergence rate. Indeed, as it follows from the next theorem the convergence to zero in (4.30) may be arbitrary slow.

Theorem 4.4 ([13, Theorem 3.4]) Let $\delta_{n}>0$ be a sequence with $\delta_{n} \rightarrow 0$ as $n \rightarrow \infty$. Then there exists a continuous function $q:[0,1] \rightarrow \mathbb{R}$ such that

$$
\sup _{\tau \geq 0}\left\|e^{-\tau\left(D_{0}+Q\right)}-\left(e^{-\tau D_{0} / n} e^{-\tau Q / n}\right)^{n}\right\|_{\mathscr{B}\left(L^{p}([0,1])\right)}=\omega\left(\delta_{n}\right),
$$

as $n \rightarrow \infty$, where $\omega$ is the Landau symbol defined in the Introduction.

Our final comment concerns the case when $q$ is only measurable. Then it can happen that the Trotter product formula for that pair $\left\{D_{0}, Q\right\}$ does not converge in the operator-norm topology:

Theorem 4.5 ([13, Theorem 3.5]) There is a non-negative measurable function $q \in L^{\infty}([0,1])$, such that

$$
\liminf _{n \rightarrow \infty} \sup _{\tau \geq 0}\left\|e^{-\tau\left(D_{0}+Q\right)}-\left(e^{-\tau D_{0} / n} e^{-\tau Q / n}\right)^{n}\right\|_{\mathscr{B}\left(L^{p}([0,1])\right)}>0 .
$$

We note that Theorem 4.5 does not exclude the convergence of the Trotter product formula for the pair $\left\{D_{0}, Q\right\}$ in the strong operator topology.

\subsection{Comments}

Section 4.1

Our example can be considered as a kind of solvable model. It fits into the evolution cases considered in Sections 2.3 and 3.2. Indeed, one has to set $\mathfrak{X}=\mathbb{C}, p=2$, $L^{p}([0, T], \mathfrak{X})=L^{2}([0, T]), A=I$ and $B(t)=q(t)$ and $\mathscr{B}=Q$. One easily checks that

$$
\left(e^{-\tau / n \mathscr{K} / 0} e^{-\tau \mathscr{B} / n}\right)^{n}=e^{-\tau}\left(e^{-\tau / n D_{0}} e^{-\tau Q / n}\right)^{n}
$$

which shows that the convergence rate of $\left(e^{-\tau / n D_{0}} e^{-\tau Q / n}\right)^{n}$ coincides with that one $\left(e^{-\tau / n \mathscr{K}} 0 e^{-\tau \mathscr{B} / n}\right)^{n}$. One easily checks that the choice $A=I, B(t)=q(t), q(t) \geq 0$, guarantees the assumptions (A1)-(A4) If $q \in C^{0, \beta}([0, T])$, then the assumption (A5) 
is satisfied. If $Q$ is Lipschitz continuous, i.e. $q \in C^{0,1}([0, T])$, then the assumption (A6) is valid.

\section{Section 4.2}

Theorem 4.2 shows that for $\beta \in(0,1)$ the convergence rate is $O\left(1 / n^{\beta}\right)$, which is better than the convergence rates $O\left(1 / n^{\beta-\alpha}\right)$ and $O\left(1 / n^{1-\alpha}\right)$ in Theorems 3.4 and 3.5. Hence, they are not sharp.

Theorems 4.3 and 4.4 demonstrate that the convergence rate can be arbitrary slow if the smoothness of $Q$ is weaker and weaker.

Finally, Theorem 4.5 shows that there is a bounded operator such that the Trotter product formula does not converge in the operator norm. This makes clear that Theorem 3.2 becomes false if the condition that $A$ is a holomorphic generator is dropped. Indeed the operator $D_{0}$ which plays the role of $A$ of Theorem 3.2 is not a generator of holomorphic semigroup.

\section{References}

1. V. Cachia, H. Neidhardt, and V. A. Zagrebnov. Accretive perturbations and error estimates for the Trotter product formula. Integral Equations Operator Theory, 39(4):396-412, 2001.

2. V, Cachia, H. Neidhardt, and V. A. Zagrebnov. Comments on the Trotter product formula error-bound estimates for nonself-adjoint semigroups. Integral Equations Operator Theory, 42(4):425-448, 2002.

3. V. Cachia and V. A. Zagrebnov. Operator-norm approximation of semigroups by quasisectorial contractions. J. Funct. Anal., 180(1):176-194, 2001.

4. V. Cachia, V.A. Zagrebnov Operator-norm convergence of the Trotter product formula for sectorial generators. Lett. Math. Phys. 50: 203-211, 1999.

5. V. Cachia and V. A. Zagrebnov. Operator-norm convergence of the Trotter product formula for holomorphic semigroups. J. Operator Theory, 46(1):199-213, 2001.

6. T. Ichinose, H. Neidhardt, and V. A. Zagrebnov. Trotter-Kato product formula and fractional powers of self-adjoint generators. J. Funct. Anal., 207(1):33-57, 2004.

7. T. Ichinose and $\mathrm{H}$. Tamura. Error estimate in operator norm of exponential product formulas for propagators of parabolic evolution equations. Osaka J. Math., 35(4):751-770, 1998.

8. T. Ichinose, Hideo Tamura, Hiroshi Tamura, and V. A. Zagrebnov. Note on the paper: "The norm convergence of the Trotter-Kato product formula with error bound" by T. Ichinose and H. Tamura. Comm. Math. Phys., 221(3):499-510, 2001.

9. T. Kato. On the Trotter-Lie product formula. Proc. Japan Acad., 50:694-698, 1974.

10. T. Kato. Trotter's product formula for an arbitrary pair of self-adjoint contraction semigroups. In Topics in functional analysis (essays dedicated to M. G. Krĕn on the occasion of his 70th birthday), volume 3 of Adv. in Math. Suppl. Stud., pages 185-195. Academic Press, New York-London, 1978.

11. T. Kato. Perturbation theory for linear operators. Classics in Mathematics. Springer-Verlag, Berlin, 1995. Reprint of the 1980 edition.

12. H. Neidhardt, A. Stephan, and V. A. Zagrebnov. Convergence rate estimates for Trotter product approximations of solution operators for non-autonomous Cauchy problems. arXiv:1612.06147 [math.FA], December 2016.

13. H. Neidhardt, A. Stephan, and V. A. Zagrebnov. Remarks on the operator-norm convergence of the Trotter product formula. ArXiv 1703.09536 [math-ph], March 2017.

14. H. Neidhardt, A. Stephan, and V.A. Zagrebnov. On convergence rate estimates for approximations of solution operators for linear non-autonomous evolution equations. Nanosyst., Phys. Chem. Math., 8(2):202-215, 2017. 
15. H. Neidhardt and V. A. Zagrebnov. On error estimates for the Trotter-Kato product formula. Lett. Math. Phys., 44(3):169-186, 1998.

16. H. Neidhardt and V. A. Zagrebnov. Trotter-Kato product formula and operator-norm convergence. Comm. Math. Phys., 205(1):129-159, 1999.

17. H. Neidhardt. On abstract linear evolution equations. I. Math. Nachr., 103:283-298, 1981.

18. H. Neidhardt. On abstract linear evolution equations. II. Prepr., Akad. Wiss. DDR, Inst. Math. P-MATH-07/81, 56 p. (1981)., 1981.

19. H. Neidhardt. On linear evolution equations. III: Hyperbolic case. Prepr., Akad. Wiss. DDR, Inst. Math. p-MATH-05/82, 74 p. (1982)., 1982.

20. H. Neidhardt and V. A. Zagrebnov. Fractional powers of self-adjoint operators and TrotterKato product formula. Integral Equations Operator Theory, 35(2):209-231, 1999.

21. H. Neidhardt and V. A. Zagrebnov. Linear non-autonomous Cauchy problems and evolution semigroups. Adv. Differential Equations, 14(3-4):289-340, 2009.

22. G. Nickel. On evolution semigroups and nonautonomous Cauchy problems. Diss. Summ. Math., 1(1-2):195-202, 1996.

23. Dzh. L. Rogava. Error bounds for Trotter-type formulas for self-adjoint operators, Funct. Anal. Appl. 27: 217-219, 1993

24. H.Tamura. A remark on operator-norm convergence of Trotter-Kato product formula. Integral Equations Operator Theory, 37(3):350-356, 2000.

25. H. F. Trotter. On the product of semi-groups of operators. Proc. Amer. Math. Soc., 10:545-551, 1959. 